PROCEEDINGS OF THE

AMERICAN MATHEMATICAL SOCIETY

Volume 132, Number 1, Pages 15-31

S 0002-9939(03)07040-0

Article electronically published on May 8, 2003

\title{
p-ADIC FORMAL SERIES AND PRIMITIVE POLYNOMIALS OVER FINITE FIELDS
}

\author{
SHUQIN FAN AND WENBAO HAN
}

(Communicated by Wen-Ching Winnie Li)

\begin{abstract}
In this paper, we investigate the Hansen-Mullen conjecture with the help of some formal series similar to the Artin-Hasse exponential series over $p$-adic number fields and the estimates of character sums over Galois rings. Given $n$ we prove, for large enough $q$, the Hansen-Mullen conjecture that there exists a primitive polynomial $f(x)=x^{n}-a_{1} x^{n-1}+\cdots+(-1)^{n} a_{n}$ over $F_{q}$ of degree $n$ with the $m$-th $(0<m<n)$ coefficient $a_{m}$ fixed in advance except when $m=\frac{n+1}{2}$ if $n$ is odd and when $m=\frac{n}{2}, \frac{n}{2}+1$ if $n$ is even.
\end{abstract}

\section{INTRODUCTION}

Let $F_{q}$ be a finite field with $q=p^{k}$ elements where $p$ is a prime number, and let $k$ a positive integer. A monic polynomial $f(x) \in F_{q}[x]$ of degree $n$ is called a primitive polynomial if the least positive integer $T$ such that $f(x) \mid x^{T}-1$ over $F_{q}[x]$ is $q^{n}-1$. Hansen and Mullen [9] conjectured that with the three nontrivial exceptions

$$
(q, n, m, a)=(4,3,1,0),(4,3,2,0),(2,4,2,1),
$$

there exists a primitive polynomial over $F_{q}$ of degree $n$ with the $m$-th $(0<m<n)$ coefficient $a \in F_{q}$ fixed in advance. For $m=1$, the Hansen-Mullen conjecture is true by the work on the existence of primitive element solutions of trace equations over finite fields, which was done by Cohen 11, Davenport [3, Jungnickel and Vanstone [1], Moreno [17], etc. For $m=2$, Han [5, 6] proved that the Hansen-Mullen conjecture is true if

(1) $q$ is odd and $n \geq 7$; or

(2) $q$ is even and $(n, a) \neq(4,0),(5,0),(6,0)$.

On the other hand, Han [7] discussed Cohen's Problem 2$]$ and proved that for any positive integer $k<p$, there exists a primitive polynomial $f(x)=x^{n}-a_{1} x^{n-1}+$ $\cdots+(-1)^{n} a_{n}$ over $F_{q}$ of degree $n$ with the first $k$ coefficients $a_{1}, a_{2}, \cdots, a_{k}$ fixed in advance if $n>2 k$ and $q$ is large enough. As a corollary, the Hansen-Mullen conjecture of the $k$-th $(1 \leq k<p)$ coefficient is true for $q$ large enough and $n>2 k$. The main idea of the proof is to express the conditions on the coefficients of the primitive polynomial in terms of traces of powers of its roots.

Received by the editors March 13, 2002 and, in revised form, August 24, 2002.

2000 Mathematics Subject Classification. Primary 11T55, 11F85, 11L40, 11L07.

Key words and phrases. Finite field, primitive polynomial, character sums over Galois rings, $p$-adic formal series.

This work was supported by NSF of China with contract No. 19971096 and No. 90104035. 
For $k \geq p$, however, we must cope with the inevitable problems relating to the characteristic in handling the trace conditions. In this paper, we will transfer the working to the unramified extensions of the $p$-adic fields and their completions, as well as the appropriate quotient rings, Galois rings where we can translate the existence of the primitive polynomial into the existence of the primitive element solution of some system of trace equations over Galois rings. To estimate the number of primitive element solutions, we need the estimates of character sums over Galois rings [10, 13 and over the Teichmüller points of $p$-adic number fields [15].

In this paper, we investigate the Hansen-Mullen conjecture with the help of some $p$-adic formal series similar to the Artin-Hasse exponential series over $K_{k}$ (the unique unramified extension of $\mathbb{Q}_{p}$ of degree $k$ ) and the estimates of character sums over Galois rings. The paper is arranged as follows. First we give a short review on character sums over Galois rings. In Section 3, we give a description of the HansenMullen conjecture over $O_{k}$ (the ring of integers of $K_{k}$ ). Then we define a $p$-adic formal series $E_{t, e}(x)$ over $K_{k}$ associated with the traces of powers of Teichmüller points $\xi \in \Gamma_{n k}$, the set of Teichmüller points in $K_{n k}$. Using Dieudonné's Theorem we prove that $E_{t, e}(x) \in O_{k}[[x]]$ so that we obtain a congruence connection between the traces of powers of Teichmüller points and the coefficients of the lifting primitive polynomials over $O_{k}$. In Section 4, we obtain the estimate of the number of the primitive element solutions in $\Gamma_{n k}$ of some system of trace equations by using the estimates of character sums over Galois rings. In fact, we get two main results: (a) The Hansen-Mullen conjecture of the $m$-th $(0<m<n)$ coefficient over $F_{q}$ holds if $q^{\frac{n}{2}-\left(m-\left[\frac{m_{1}}{p}\right]\right)}>m 2^{\omega\left(q^{n}-1\right)}$; (b) The Hansen-Mullen conjecture of the $(n-m)$-th $(0<m<n)$ coefficient over $F_{q}$ holds if $q^{\frac{n}{2}-\left(m-\left[\frac{m_{1}}{p}\right]\right)-1}>m 2^{\omega\left(q^{n}-1\right)}$. In the above, $\omega\left(q^{n}-1\right)$ is the number of the distinct prime factors of $q^{n}-1$ and $m_{1}$ is the "non- $p$ part" of $m$, i.e. $m_{1}$ is the largest divisor of $m$ prime to $p$. In fact, (a) and (b) are suitable for those $m$ such that $m \leq \frac{n}{2}$ and $m>\frac{n}{2}$, respectively. As an asymptotic result, we prove that for any given $n$, there exists a constant $c(n)$ such that the Hansen-Mullen conjecture over $F_{q}$ of the $m$-th coefficient is true for $q>c(n)$ except when $m=\frac{n+1}{2}$ if $n$ is odd and when $m=\frac{n}{2}, \frac{n}{2}+1$ if $n$ is even.

\section{Estimates of CHARACTER sums OVER GALOIS RINGS}

2.1. $p$-adic number fields and Galois rings. Let $p$ be a prime number. For $r=\frac{a}{b} \in \mathbb{Q}, a, b \in \mathbb{Z} \backslash\{0\}$, define the order of $a \in \mathbb{Z}$ at $p$, denoted by $\operatorname{ord}_{p} a$, to be the largest integer $d$ such $p^{d} \mid a$ and $\operatorname{ord}_{p} r=\operatorname{ord}_{p} a-\operatorname{ord}_{p} b$. The non-archimedean valuation ||$_{p}$ on $\mathbb{Q}$ can be defined by

$$
\left\{\begin{array}{l}
|0|_{p}=0 \\
|r|_{p}=p^{- \text {ord }_{p} r} .
\end{array}\right.
$$

It is well known that ||$_{p}$ is a metric on $\mathbb{Q}$.

Let $\mathbb{Q}_{p}$ be the completion of $\mathbb{Q}$ with respect to the metric ||$_{p}$, let $K_{k}$ be the unique unramified extension of $\mathbb{Q}_{p}$ of degree $k$, let $O_{k}=\left\{x \in K_{k} \|\left. x\right|_{p} \leq 1\right\}$ be the ring of integers of $K_{k}$, let $\overline{\mathbb{Q}_{p}}$ be the algebraic closure of $\mathbb{Q}_{p}$, and let $\Omega$ be the completion of $\overline{\mathbb{Q}_{p}}$. Denote the set of the Teichmüller points in $K_{k}$ by

$$
\Gamma_{k}=\left\{\xi \in K_{k} \mid \xi^{p^{k}}=\xi\right\}
$$


and $\Gamma_{k}^{*}=\Gamma_{k} \backslash\{0\}$. Then every element $\alpha \in K_{k}$ can be written in a unique way as

$$
\alpha=\sum_{i=i_{0}}^{\infty} a_{i} p^{i} \quad \text { where } a_{i} \in \Gamma_{k}, i_{0} \in \mathbb{Z}
$$

If $\alpha \in O_{k}$, we have

$$
\alpha=\sum_{i=0}^{\infty} a_{i} p^{i} \quad \text { where } a_{i} \in \Gamma_{k} .
$$

Define the canonical projective map $\phi$ from $O_{k}$ to $\Gamma_{k}$ by

$$
\phi(\alpha)=a_{0} .
$$

In fact, $O_{k}$ is a local ring with unique maximal ideal $P_{k}=p O_{k}$. For $e \geq 1$, the Galois ring $R_{e, k}$ is defined by $O_{k} / p^{e} O_{k}$. In particular, when $e=1, R_{e, k}=F_{q}$ is a finite field with $q=p^{k}$ elements and $F_{q}=\left\{\bar{\xi} \mid \xi \in \Gamma_{k}\right\}$ where $\bar{\xi}$ is the residue class $\bmod p$ including $\xi$. It is obvious that any element $\beta \in R_{e, k}$ can be uniquely written as

$$
\beta=\sum_{i=0}^{e-1} b_{i} p^{i} \quad \text { where } b_{i} \in \Gamma_{k} .
$$

Let $n>0$ be an integer and $\tau_{k}$ the Frobenius map of $K_{n k}$ over $K_{k}$ given by

$$
\tau_{k}(z)=\sum_{i=i_{0}}^{\infty} a_{i}^{p^{k}} p^{i}
$$

for $z=\sum_{i=i_{0}}^{\infty} a_{i} p^{i} \in K_{n k}$, where $a_{i} \in \Gamma_{n k}, i_{0} \in \mathbb{Z}$. As we know, $\tau_{k}$ is the generator of the Galois group of $K_{n k} / K_{k}$ which is a cyclic group of order $n$. The trace map $\operatorname{Tr}(\cdot): K_{n k} \longrightarrow K_{k}$ is defined via

$$
\operatorname{Tr}(x)=x+\tau_{k}(x)+\cdots+\tau_{k}^{n-1}(x)
$$

for $x \in K_{n k}$.

$\left.\tau_{k}\right|_{O_{n k}} \bmod p^{e}$ is the Frobenius map of $R_{e, n k}$ over $R_{e, k}$. Later we also use $\tau_{k}$ to denote $\left.\tau_{k}\right|_{O_{n k}} \bmod p^{e}$ without confusion. As we know, $\tau_{k}$ is the generator of the Galois group of $R_{e, n k} / R_{e, k}$ which is a cyclic group of order $n$. More precisely, we have

$$
\tau_{k}(z)=\sum_{i=0}^{e-1} a_{i}^{p^{k}} p^{i}
$$

for $z=\sum_{i=0}^{e-1} a_{i} p^{i} \in R_{e, n k}$, where $a_{i} \in \Gamma_{n k}, i=0,1, \cdots, e-1$.

The map $\operatorname{Tr}_{e, n k, k}(\cdot)=\left.\operatorname{Tr}(\cdot)\right|_{O_{n k}} \bmod p^{e}$ is the trace map from $R_{e, n k}$ to $R_{e, k}$. More precisely,

$$
\operatorname{Tr}_{e, n k, k}(x)=x+\tau_{k}(x)+\cdots+\tau_{k}^{n-1}(x)
$$

for $x \in R_{e, n k}$.

Similarly, the norm map $N \operatorname{rom}(\cdot)$ from $K_{n k}$ to $K_{k}$ can be defined by

$$
\operatorname{Norm}(x)=x \cdot \tau_{k}(x) \cdots \tau_{k}^{n-1}(x)
$$

for $x \in K_{n k}$. 
2.2. Characters over Galois rings. Let $e, k, n \in \mathbb{Z}_{>0}$. Now we give a few basic facts on the additive characters over Galois rings $R_{e, k}$ and multiplicative characters over $\Gamma_{n k}^{*}$.

2.2.1. Additive characters over Galois rings. An additive character of $R_{e, k}$ is a homomorphism from the additive group of $R_{e, k}$ to $\mathbb{C}^{*}$, the multiplicative group of a complex field. Define $\psi(c)=e^{2 \pi i T r_{e, k, 1}(c) / p^{e}}$ for $c \in R_{e, k}$. It is easily seen that $\psi$ is an additive character of $R_{e, k}$, that is, the so-called canonical additive character. For $a \in R_{e, k}$, define $\psi_{a}(c)=\psi(a c), c \in R_{e, k}$. Similar to the case of finite fields, we can prove that $\psi_{a}$ is also an additive character. In fact, we have

Lemma 1. $\left\{\psi_{a}\right\}_{a \in R_{e, k}}$ consists of all the additive characters of $R_{e, k}$.

Proof. It is obvious that we only need to prove $a=0$ if and only if $\psi_{a}$ is trivial, that is, a principle character. Suppose $a \neq 0, a=p^{l} u$, where $u \in R_{e, k}^{*}, 0 \leq l \leq e-1$, such that $\operatorname{Tr}_{e, k, 1}(a c)=0$ for all $c \in R_{e, k}$. We have $p^{l} \operatorname{Tr}_{e, k, 1}(u c)=0$ for all $c \in R_{e, k}$, hence $p^{l} \operatorname{Tr}_{e, k, 1}(c)=0$ for all $c \in R_{e, k}$. Since $\operatorname{Tr}_{e, k, 1}(\cdot): R_{e, k} \rightarrow \mathbb{Z}_{p^{e}}$ is surjective, there exists $c^{\prime} \in R_{e, k}$ such that $\operatorname{Tr}_{e, k, 1}\left(c^{\prime}\right)=1$. This gives $p^{l}=0$, a contradiction.

Lemma 2. Let $a \in R_{e, k}$, and let $\psi$ be the canonical additive character of $R_{e, k}$. We have

$$
\sum_{c \in R_{e, k}} \psi_{c}(a)=\left\{\begin{array}{cl}
q^{e} & \text { if } a=0 \\
0 & \text { if } a \neq 0
\end{array}\right.
$$

Proof. Special case for Theorem 5.4 in [16].

We have a more general result than Lemma 2

Lemma 3. Let $a \in R_{e, k}$, and let $\psi$ be the canonical additive character of $R_{e, k}$. We have

$$
\sum_{c \in R_{d, k}} \psi_{c}\left(p^{e-d} a\right)= \begin{cases}q^{d} & \text { if } a=0 \bmod p^{d} \\ 0 & \text { otherwise }\end{cases}
$$

where $1 \leq d \leq e$.

2.2.2. Multiplicative character over Teichmüller points. By definition, $\Gamma_{n k}$ is the set of Teichmüller points in $K_{n k}$ and is independent on $e . \Gamma_{n k}^{*}$ forms a multiplicative group with order $q^{n}-1$. Let $g$ be a primitive element (i.e. generator) of $\Gamma_{n k}^{*}$; the canonical multiplicative character $\chi$ can be defined by $\chi\left(g^{l}\right)=e^{2 \pi i l / q^{n}-1}$ for $0 \leq l \leq q^{n}-2$. For $0 \leq j \leq q^{n}-2$, define $\chi_{j}\left(g^{l}\right)=\chi\left(g^{l j}\right)$. The $\chi_{j}$ 's are all the multiplicative characters of $\Gamma_{n k}^{*}$ and form a cyclic group with order $q^{n}-1$. It is familiar that the order of each character $\chi_{j}$ is a divisor of $q^{n}-1$.

Lemma 4. Let $n$ be a positive integer, $\xi \in \Gamma_{n k}^{*}$. Then we have

$$
\sum_{d \mid q^{n}-1} \frac{\mu(d)}{\varphi(d)} \sum_{\chi^{(d)}} \chi^{(d)}(\xi)= \begin{cases}\frac{q^{n}-1}{\varphi\left(q^{n}-1\right)} & \text { if } \xi \text { is a primitive element of } \Gamma_{n k}^{*} \\ 0 & \text { otherwise, }\end{cases}
$$

where $\mu(d)$ is the Möbius function and $\varphi(d)$ is the Euler function, and $\chi^{(d)}$ runs through all the $\varphi(d)$ multiplicative characters over $\Gamma_{n k}^{*}$ with order $d$. 
Proof. In the following formula, $\gamma$ runs through all the distinct prime factors of $q^{n}-1$ :

$$
\sum_{d \mid q^{n}-1} \frac{\mu(d)}{\varphi(d)} \sum_{\chi^{(d)}} \chi^{(d)}(\xi)=\prod_{\gamma \mid q^{n}-1}\left(1+\frac{\mu(\gamma)}{\varphi(\gamma)} \sum_{\chi^{(\gamma)}} \chi^{(\gamma)}(\xi)\right) .
$$

If $\xi$ is a primitive element of $\Gamma_{n k}^{*}$, then

$$
\prod_{\gamma \mid q^{n}-1}\left(1+\frac{\mu(\gamma)}{\varphi(\gamma)} \sum_{\chi^{(\gamma)}} \chi^{(\gamma)}(\xi)\right)=\prod_{\gamma \mid q^{n}-1}\left(1+\frac{1}{\varphi(\gamma)}\right)=\frac{q^{n}-1}{\varphi\left(q^{n}-1\right)} .
$$

Otherwise, there exists a prime number $\gamma \mid \frac{q^{n}-1}{\operatorname{order}(\xi)}$ so that $\sum_{\chi^{(\gamma)}} \chi^{(\gamma)}(\xi)=\varphi(\gamma)$, hence

$$
\prod_{\gamma \mid q^{n}-1}\left(1+\frac{\mu(\gamma)}{\varphi(\gamma)} \sum_{\chi^{(\gamma)}} \chi^{(\gamma)}(\xi)\right)=0
$$

2.3. Estimates of character sums over Galois rings. Let $k, n \geq 1$, and let $h(x)$ be a polynomial over $R_{e, n k}$ with $h(0)=0$ and $h(x)$ not identically 0 . Let

$$
h(x)=h_{0}(x)+h_{1}(x) p+\cdots+h_{e-1}(x) p^{e-1}, \quad h_{i}(x) \in \Gamma_{n k}[x],
$$

be the $p$-adic expansion of $h(x)$. Such an expansion can be derived from a $p$-adic expansion of the coefficients of $h(x)$. Let $d_{i}$ be the degree of $h_{i}(x)$ and let

$$
h_{i}(x)=\sum_{j=0}^{d_{i}} h_{i, j} x^{j}, \quad h_{i, j} \in \Gamma_{n k} .
$$

$h(x)$ is called nondegenerate if the coefficients $h_{i, j}$ of $h_{i}(x)$ satisfy

$$
h_{i, j}=0, \quad \text { if } j \equiv 0(\bmod p), 0 \leq j \leq d_{i}, 0 \leq i \leq e-1 .
$$

Define the weighted e-degree of $h(x)$ by

$$
D_{e}(h(x))=\max \left(d_{0} p^{e-1}, d_{1} p^{e-2}, \cdots, d_{e-1}\right) .
$$

Various kinds of character sums over Galois rings are investigated; see for example [10], [15], etc. Here we give two theorems from [15] in a little different form for our later use. These results are analogous to Weil estimates on character sums over finite fields.

Theorem 5 ([13], [15]). Let $f(x) \in R_{e, n k}[x]$ be nondegenerate with weighted edegree $D_{e}(f(x))$, and let $\psi_{e, n}$ be a nontrivial additive character of $R_{e, n k}$. Then

$$
\left|\sum_{\xi \in \Gamma_{n k}} \psi_{e, n}(f(\xi))\right| \leq\left(D_{e}(f(x))-1\right) q^{n / 2} .
$$

For twisted character sums, we have

Theorem 6 ([15]). Let $f(x) \in R_{e, n k}[x]$ be nondegenerate with weighted e-degree $D_{e}(f(x)), \psi_{e, n}$ a nontrivial additive character of $R_{e, n k}$ and $\chi$ a nontrivial multiplicative character of $\Gamma_{n k}^{*}$. Then

$$
\left|\sum_{\xi \in \Gamma_{n k}^{*}} \psi_{e, n}(f(\xi)) \chi(\xi)\right| \leq D_{e}(f(x)) q^{n / 2} .
$$




\section{HANSEn-Mullen CONJECTURE OVER $p$-ADiC NUMBER FIELDS}

Throughout this section, $q=p^{k}, e \in \mathbb{Z}_{>0}$.

Let $\widetilde{f}(x) \in O_{k}[x]$ be a monic polynomial of degree $n$. We call $\tilde{f}(x)$ a basic irreducible polynomial over $O_{k}$ if $\widetilde{f}(x) \bmod p$ is irreducible over $F_{q}$.

Definition 7. Let $\widetilde{f}(x) \in O_{k}[x]$ be a basic irreducible polynomial of degree $n$. We call $\tilde{f}(x)$ a lifting primitive polynomial over $O_{k}$ if there exists a positive integer $T$ such that $\tilde{f}(x) \mid x^{T}-1$ and the least positive integer $T=q^{n}-1$.

In fact, the set of primitive elements of $\Gamma_{n k}^{*}$ (all the generators of $\Gamma_{n k}^{*}$ as a cyclic multiplicative group) is the same as the set of roots (in $O_{n k}$ ) of lifting primitive polynomials of degree $n$ in $O_{k}[x]$. So in the rest of the paper, we will identify them without explanation.

If $\widetilde{f}(x)$ is a lifting primitive polynomial of $O_{k}[x]$, it is easily seen that $f(x)=\tilde{f}(x)$ $\bmod p$ is a primitive polynomial over $F_{q}$. On the other hand, if $f(x)$ is a primitive polynomial over $F_{q}$, by the Hensel Lemma there is a polynomial $\tilde{f}(x) \in O_{k}[x]$ such that $f(x) \equiv \tilde{f}(x) \bmod p$ and $\tilde{f}(x)$ is a lifting primitive polynomial over $O_{k}$.

By the discussions above, the coefficients of primitive polynomials over $F_{q}$ and the lifting primitive polynomials over $O_{k}$ are closely related. Now we reformulate the Hansen-Mullen conjecture using $O_{k}$.

Hansen-Mullen conjecture over $O_{k}$. Let $q=p^{k}$. For any given $a \in \Gamma_{k}$, with the three nontrivial exceptions

$$
(q, n, m, a)=(4,3,1,0),(4,3,2,0),(2,4,2,1),
$$

there exists a lifting primitive polynomial $f(x)$ over $O_{k}$ of degree $n$ with the $m$-th $(0<m<n)$ coefficient $a_{m}$ fixed in advance such that $\phi\left(a_{m}\right)=a$, where $\phi$ is the canonical projective map from $O_{k}$ to $R_{1, k}=F_{q}$.

So we only need to consider the existence of the lifting primitive polynomials over $O_{k}$. As in [5, 6, 7], we want to express the conditions on the coefficients of a primitive polynomial over $F_{q}$ in terms of traces of the powers of the roots in an extension field of $F_{q}$ of the primitive polynomial. To cope with the inevitable problems relating to the characteristic in handling the trace conditions, the work is transferred to the unramified extensions of the $p$-adic field and their completions, as well as the appropriate quotient rings, Galois rings.

For this reason, we investigate the relation between the coefficients of the lifting primitive polynomial over $p$-adic number field $K_{k}$ and the traces of the powers of the roots, in the extension field $K_{n k}$, of the lifting primitive polynomial and reducing the formula associated to the coefficients and the roots modulo suitable $p^{e}$.

Lemma 8 ([12]). Let $A$ be an $n \times n$ matrix with entries in $\Omega$. We have the following identity of formal power series in $\Omega[x]$ :

$$
\operatorname{det}(1-A x)=\exp \left(-\sum_{s=1}^{\infty} \operatorname{Tr}\left(A^{s}\right) x^{s} / s\right)
$$

where $\operatorname{Tr}\left(A^{s}\right)$ is the trace of matrix $A^{s}, s=1,2, \cdots$.

Let $\tilde{f}(x)$ be a lifting primitive polynomial of degree $n$ over $O_{k}$. Now we give the relation between the coefficients of lifting primitive polynomial $\tilde{f}(x)$ over $p$-adic number field $K_{k}$ and the traces of the powers of the roots of $\widetilde{f}(x)$ in the extension 
field $K_{n k}$. It is easy to see that if $\xi$ is a root of $\widetilde{f}(x)$ in $\Omega$, then $\xi, \xi^{q}, \cdots, \xi^{q^{n-1}}$ are all the roots of $\widetilde{f}(x)$ in $\Omega$.

Lemma 9. Let $\widetilde{f}(x)=x^{n}-\sigma_{1} x^{n-1}+\cdots+(-1)^{n} \sigma_{n} \in O_{k}[x]$ be a lifting primitive polynomial of degree $n$, and let $\xi$ be a root of $\widetilde{f}(x)$ in $\Omega$. We have

$$
x^{n} \widetilde{f}\left(\frac{1}{x}\right)=\exp \left(-\sum_{s=1}^{\infty} \operatorname{Tr}\left(\xi^{s}\right) x^{s} / s\right) .
$$

Proof. Let $A=\left(a_{i j}\right)_{1 \leq i \leq n, 1 \leq j \leq n}$, where

$$
a_{i j}= \begin{cases}0 & \text { if } i \neq j ; \\ \xi^{q^{i-1}} & \text { if } i=j .\end{cases}
$$

Expanding the left-hand and right-hand sides of (11), respectively, we get (2).

Now we go back to the Hansen-Mullen conjecture and discuss the relation between the existence of lifting primitive polynomials of degree $n$ over $O_{k}$ with the $m$-th coefficient fixed for $1 \leq m \leq n-1, m=p^{e-1} m_{1},\left(m_{1}, p\right)=1$ and the existence of primitive element solutions in $\Gamma_{n k}^{*}$ of some kind of system of trace equations, that is:

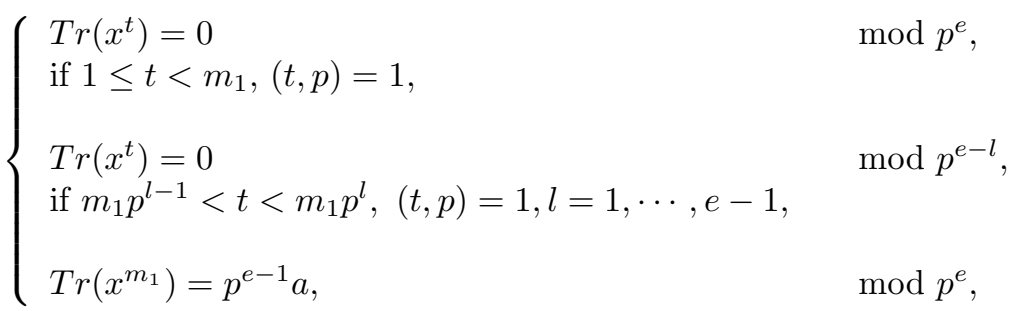

where $\operatorname{Tr}(\cdot)$ is the trace from $K_{n k}$ to $K_{k}, a \in \Gamma_{k}$.

Theorem 10. Let $a \in \Gamma_{k}, m=p^{e-1} m_{1},\left(m_{1}, p\right)=1$ and $e \geq 1$. Let

$$
\begin{aligned}
\widetilde{f}(x) & =(x-\xi)\left(x-\xi^{q}\right) \cdots\left(x-\xi^{q^{n-1}}\right) \\
& =x^{n}-\sigma_{1} x^{n-1}+\cdots+(-1)^{n} \sigma_{n}
\end{aligned}
$$

be the minimal polynomial of $\xi$ over $O_{k}$ where the system of equations (3) is assumed to have a primitive element solution $\xi \in \Gamma_{n k}^{*}$. For $1 \leq m \leq n$, we have

$$
\sigma_{m} \equiv(-1)^{m-1} m_{1}^{-1} a^{p^{e-1}} \bmod p .
$$

Proposition 11. If the system of equations (3) has one primitive element solution $\xi \in \Gamma_{n k}^{*}$ for any given $a \in \Gamma_{k}$, the Hansen-Mullen conjecture is true for the $m$-th coefficient.

Proof. By Theorem 10 $\phi\left(\sigma_{m}\right)$ runs across $\Gamma_{k}$ if $a$ runs through $\Gamma_{k}$.

Since the reciprocal polynomial of a (lifting) primitive polynomial over $F_{q}$ (or $O_{k}$ ) is also a (lifting) primitive polynomial, then, if there exists a (lifting) primitive polynomial over $F_{q}$ (or $O_{k}$ ) with the $m$-th coefficient fixed and the constant term fixed as a primitive element $b$ in $\Gamma_{k}$, there exists a primitive polynomial with $(n-m)$ th coefficient fixed. So we consider another system of equations with the constant 
term fixed:

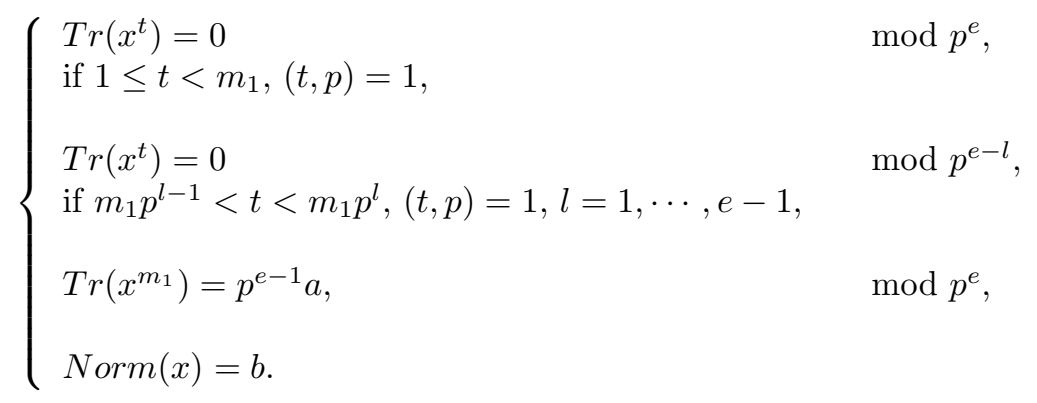

Proposition 12. Let $b$ be a primitive element of $\Gamma_{k}$. If the system of equations (41) has one primitive element solution $\xi \in \Gamma_{n k}^{*}$ for any given $a \in \Gamma_{k}$, the HansenMullen conjecture is true for the $(n-m)$-th coefficient.

Proof. Let $\xi \in \Gamma_{n k}^{*}$ be a primitive element solution of (4), $f(x)$ the minimal polynomial of $\xi$ over $O_{k}$ and $\sigma_{m}$ the $m$-th coefficient of $f(x)$. Then $\frac{1}{b} x^{n} f\left(\frac{1}{x}\right)$ is a lifting primitive polynomial over $O_{k}$ and the $(n-m)$-th coefficient of $\frac{1}{b} x^{n} f\left(\frac{1}{x}\right)$ is $\frac{\sigma_{m}}{b}$. By Theorem 10 $\phi\left(\frac{\sigma_{m}}{b}\right)$ runs across $\Gamma_{k}$ if $a$ runs through $\Gamma_{k}$. So we finish the proof of the Hansen-Mullen conjecture for the $(n-m)$-th coefficient.

Before we prove Theorem 10, we give a simple result.

Lemma 13. Let $(t, p)=1$ and $\hat{\xi} \in \Gamma_{n k}$. We have

$$
\operatorname{Tr}\left(\hat{\xi}^{t p^{i}}\right)=\tau^{i}\left(\operatorname{Tr}\left(\hat{\xi}^{t}\right)\right)
$$

where $\tau^{i}$ is the Frobenius map of $K_{k}$ over $Q_{p}$ for $i=0,1, \cdots$.

To prove Theorem 10, we define, for later use, a formal series over $K_{k}$ similar to the Artin-Hasse exponential series associated with the trace of power of $\xi \in \Gamma_{n k}^{*}$, that is,

$$
E_{t, e}(x)=\exp \left(-\sum_{s=e}^{\infty} \frac{\operatorname{Tr}\left(\xi^{t p^{s}}\right) x^{t p^{s}}}{t p^{s}}\right)
$$

where $t, e \in \mathbb{Z}_{\geq 0}$ and $(t, p)=1$. Later we use $E_{t}$ to denote $E_{t, 0}$. We want to discuss the $p$-adic property of the coefficients of $E_{t, e}(x)$ under some conditions. Now we give Dieudonné's Theorem as a lemma.

Lemma 14 (Dieudonné's Theorem). Let $f(x)=1+a_{1} x+a_{2} x^{2}+\cdots \in 1+x K_{k}[[x]]$. Then

$$
f(x) \in 1+x O_{k}[[x]]
$$

if and only if

$$
\frac{f(x)^{p}}{f^{\tau}\left(x^{p}\right)} \in 1+p x O_{k}[[x]]
$$

where $\tau$ is the Frobenius map of $K_{k}$ over $Q_{p}$.

Lemma 15. Let $t, e \geq 1$ be positive integers satisfying $(t, p)=1$, and let $\xi$ be a primitive element of $\Gamma_{n k}^{*}$ such that $\operatorname{Tr}\left(\xi^{t}\right) \equiv 0 \bmod p^{e}$. Then we have

$$
E_{t, e}(x) \in 1+x O_{k}[[x]] .
$$


Proof. Consider

$$
\begin{aligned}
E_{t, e}(x)^{p} & =\exp \left(-p \sum_{s=e}^{\infty} \frac{\operatorname{Tr}\left(\xi^{t p^{s}}\right)}{t p^{s}} x^{t p^{s}}\right) \\
& =\exp \left(-p \frac{\operatorname{Tr}\left(\xi^{t p^{e}}\right)}{t p^{e}} x^{t p^{e}}-p \sum_{s=e+1}^{\infty} \frac{\operatorname{Tr}\left(\xi^{t p^{s}}\right)}{t p^{s}} x^{t p^{s}}\right)
\end{aligned}
$$

and

$$
\begin{aligned}
E_{t, e}^{\tau}\left(x^{p}\right) & =\exp \left(-\sum_{s=e}^{\infty} \frac{\operatorname{Tr}\left(\xi^{t p^{s+1}}\right)}{t p^{s}} x^{t p^{s+1}}\right) \\
& =\exp \left(-p \sum_{s=e+1}^{\infty} \frac{\operatorname{Tr}\left(\xi^{t p^{s}}\right)}{t p^{s}} x^{t p^{s}}\right) .
\end{aligned}
$$

Therefore

$$
\frac{E_{t, e}(x)^{p}}{E_{t, e}^{\tau}\left(x^{p}\right)}=\exp \left(-p \frac{\operatorname{Tr}\left(\xi^{t p^{e}}\right)}{t p^{e}} x^{t p^{e}}\right) \in 1+p x O_{k}[[x]] .
$$

By Dieudonné's Theorem, we have

$$
E_{t, e}(x) \in 1+x O_{k}[[x]]
$$

Lemma 16. Let $t, e \geq 1$ be positive integers satisfying $(t, p)=1$, and let $\xi$ be a primitive element of $\Gamma_{n k}^{*}$ such $\operatorname{Tr}\left(\xi^{t}\right) \equiv 0 \bmod p^{e}$. Let

$$
E_{t}(x)=1+a_{1} x+\cdots+a_{l} x^{l}+\cdots
$$

Then we have

$$
a_{l} \in p O_{k} \quad \text { if } p^{e} \nmid l .
$$

More precisely

$$
E_{t}(x) \equiv E_{t, e}(x) \bmod p
$$

Proof. We rewrite $E_{t}(x)$ as

$$
E_{t}(x)=\exp \left(-\sum_{s=e}^{\infty} \frac{\operatorname{Tr}\left(\xi^{t p^{s}}\right)}{t p^{s}} x^{t p^{s}}\right) \prod_{i=0}^{e-1} \exp \left(-\frac{\operatorname{Tr}\left(\xi^{t p^{i}}\right)}{t p^{i}} x^{t p^{i}}\right) .
$$

Since the first term (i.e., $\left.E_{t, e}(x)\right)$ is in $1+x O_{k}[[x]]$ and the second product of the right-hand side belongs to $1+p x O_{k}[[x]]$, the only possible terms $a_{l}$ such that $a_{l} \notin p O_{k}$ are the terms in the expansion of $E_{t, e}(x)$. So we must have $p^{e} \mid l$.

Now we come back to prove Theorem 10 . 
Proof of Theorem 10, By Lemma 9, we have

$$
\begin{aligned}
x^{n} \tilde{f}\left(\frac{1}{x}\right) & =\exp \left(-\sum_{s=1}^{\infty} \operatorname{Tr}\left(\xi^{s}\right) x^{s} / s\right) \\
& =\prod_{\substack{(t, p)=1 \\
t>0}} E_{t}(x)=\prod_{\substack{(t, p)=1 \\
t \leq m}} E_{t}(x) \prod_{\substack{(t, p)=1 \\
t>m}} E_{t}(x) \\
& =\prod_{\substack{(t, p)=1 \\
t \leq m_{1}}} E_{t}(x) \prod_{l=1}^{e-1} \prod_{\substack{(t, p)=1 \\
m_{1} p^{l-1}<t<m_{1} p^{l}}} E_{t}(x) \prod_{\substack{(t, p)=1 \\
t>m}} E_{t}(x) .
\end{aligned}
$$

Then by Lemma 16

$$
\begin{gathered}
x^{n} \widetilde{f}\left(\frac{1}{x}\right)=E_{m_{1}, e-1}(x) \prod_{\substack{t, p)=1 \\
t<m_{1}}} E_{t, e}(x) \prod_{l=1}^{e-1} \prod_{\substack{(t, p)=1 \\
m_{1} p^{-1}<t<m_{1} p^{l}}} E_{t}(x) \bmod p . \\
\times E_{t, e-l}(x) \prod_{\substack{t, p)=1 \\
t>m}} E_{t} .
\end{gathered}
$$

It is obvious that the degrees of the nonconstant terms in

$$
\prod_{\substack{(t, p)=1 \\ m_{1} p^{l-1}<t<m_{1} p^{l}}} E_{t, e-l}(x)
$$

for $l=1, \cdots, e-1$ and in

$$
\prod_{\substack{(t, p)=1 \\ t>m}} E_{t}(x)
$$

are greater than $m$, hence the coefficient of $x^{m}$ in $x^{n} \widetilde{f}\left(\frac{1}{x}\right) \bmod p$ is same as the coefficient of $x^{m}$ in

$$
H(x)=E_{m_{1}, e-1}(x) \prod_{\substack{0<t<m_{1} \\(t, p)=1}} E_{t, e}(x) \bmod p .
$$

Since there exists a formal series $G(x) \in O_{k}[[x]]$ such that

$$
G\left(x^{p^{e}}\right)=\prod_{\substack{0<t<m_{1} \\(t, p)=1}} E_{t, e}(x)
$$

and $m=m_{1} p^{e-1}$, the coefficient of $x^{m}$ in $H(x)$ is the same as the coefficient of $x^{m}$ in $E_{m_{1}, e-1}(x)$. By observing that the coefficient of $x^{m}$ in $E_{m_{1}, e-1}(x)$ is $-\frac{\operatorname{Tr}\left(\xi^{m}\right)}{m}$, we have

As a result, we have

$$
(-1)^{m} \sigma_{m} \equiv-\frac{\operatorname{Tr}\left(\xi^{m}\right)}{m} \bmod p .
$$

$$
\sigma_{m} \equiv(-1)^{m-1} m_{1}^{-1} a^{p^{e-1}} \bmod p .
$$

In the next section we will estimate the number of primitive element solutions of $\Gamma_{n k}^{*}$ in (3) and (4). 


\section{Estimates AND CAlCUlations}

Now we estimate the number of primitive element solutions of (3) and (4) in $\Gamma_{n k}^{*}$, respectively. For this reason, we define

$$
S_{l}=\left\{t \mid p^{l-1} m_{1}<t<p^{l} m_{1} \text { and }(t, p)=1\right\}
$$

for $l=1,2, \cdots, e-1$ and

$$
\begin{gathered}
S_{0}=\left\{t \mid 1 \leq t \leq m_{1},(t, p)=1\right\}, \\
\mathbb{S}=\left\{\left(c_{t}\right)_{(t, p)=1} \mid c_{t} \in R_{e-l, k} \text { for } t \in S_{l}, l=0,1, \cdots, e-1\right\} .
\end{gathered}
$$

Let $W=\# \mathbb{S}$. We have $W=q^{\sum_{l=0}^{e-1}(e-l) \# S_{l}}$.

Let $N$ be the number of primitive element solutions of (3) in $\Gamma_{n k}^{*}, \psi$ the canonical additive character of $R_{e, k}, \psi_{e, n}=\psi \cdot T r_{e, n k, k}$ the canonical additive character of $R_{e, n k}$ and $\chi^{(d)}$ run through all the multiplicative character over $\Gamma_{n k}^{*}$ with order $d$. From Lemmas 2, 3 and 4 .

$$
\begin{aligned}
N= & \delta \sum_{\xi \in \Gamma_{n k}^{*}}\left(\prod_{l=0}^{e-1} \prod_{\substack{t \in S_{l} \\
t \neq m_{1}}} \sum_{c_{t} \in R_{e-l, k}} \psi\left(p^{l} c_{t} \operatorname{Tr}\left(\xi^{t}\right)\right)\right) \\
& \times\left(\sum_{c_{m_{1}} \in R_{e, k}} \psi\left(c_{m_{1}}\left(\operatorname{Tr}\left(\xi^{m_{1}}\right)-p^{e-1} a\right)\right)\right) \sum_{d \mid q^{n}-1} \frac{\mu(d)}{\varphi(d)} \sum_{\chi^{(d)}} \chi^{(d)}(\xi) \\
= & \delta \sum_{d \mid q^{n}-1} \frac{\mu(d)}{\varphi(d)} \sum_{\chi^{(d)}} \sum_{\left(c_{t}\right)} \psi\left(-p^{e-1, p)=1} c_{m_{1}} a\right) \\
& \times \Lambda\left(\left(c_{t}\right)_{(t, p)=1}, \chi^{(d)}\right),
\end{aligned}
$$

where

$$
\delta=\frac{\varphi\left(q^{n}-1\right)}{\left(q^{n}-1\right)} q^{-\sum_{l=0}^{e-1}(e-l) \# S_{l}}
$$

and

$$
\Lambda\left(\left(c_{t}\right)_{(t, p)=1}, \chi^{(d)}\right)=\sum_{\xi \in \Gamma_{n k}^{*}} \chi^{(d)}(\xi) \psi_{e, n}\left(\sum_{l=0}^{e-1} p^{l} \sum_{t \in S_{l}} c_{t} \xi^{t}\right)
$$

where $\left(c_{t}\right)_{(t, p)=1} \in \mathbb{S}$.

Denote

$$
h(x)=\sum_{l=0}^{e-1} p^{l} \sum_{t \in S_{l}} c_{t} x^{t} .
$$

It is easy to check that $h(x)$ is nondegenerate if $h(x) \neq 0$. So

$$
\Lambda\left(\left(c_{t}\right)_{(t, p)=1}, \chi^{(d)}\right)=\sum_{\xi \in \Gamma_{n k}^{*}} \chi^{(d)}(\xi) \psi_{e, n}(h(x)) .
$$

Now we estimate $N$. So

(1) Suppose $h(x)=0$. Then $c_{t}=0 \in R_{e-l, k}$ for $t \in S_{l}$, where $l=0,1, \cdots, e-1$.

(a) When $d=1, \frac{\mu(d)}{\varphi(d)}=1$ and

$$
\psi\left(p^{e-1} c_{m_{1}} a\right)=1 .
$$

$$
\Lambda\left(\left(c_{t}\right)_{(t, p)=1}, \chi^{(d)}\right)=q^{n}-1 .
$$


(b) When $d>1$,

$$
\Lambda\left(\left(c_{t}\right)_{(t, p)=1}, \chi^{(d)}\right)=0 .
$$

We have

$$
\begin{aligned}
& \left.\sum_{d \mid q^{n}-1} \frac{\mu(d)}{\varphi(d)} \sum_{\chi^{(d)}} \sum_{h(x)=0} \psi\left(p^{e-1} c_{m_{1}} a\right) \times \Lambda\left(\left(c_{t}\right)_{(t, p)=1}, \chi^{(d)}\right)\right) \\
& =q^{n}-1 .
\end{aligned}
$$

(2) Suppose $h(x) \neq 0$. Then $c_{t} \neq 0$ for some $t \in S_{l}$ and some $l$ such that $0 \leq l \leq e-1$. In this case $h(x)$ is nondegenerate.

(a) When $d=1$, by Theorem 5

$$
\begin{aligned}
\left|\Lambda\left(\left(c_{t}\right)_{(t, p)=1}, \chi^{(1)}\right)\right| & \leq\left(D_{e}(h(x))-1\right) q^{n / 2}+1 \\
& \leq D_{e}(h(x)) q^{n / 2} .
\end{aligned}
$$

(b) When $d \neq 1$, by Theorem 6

$$
\left|\Lambda\left(\left(c_{t}\right)_{(t, p)=1}, \chi^{(d)}\right)\right| \leq D_{e}(h(x)) q^{n / 2} .
$$

In the above $D_{e}(h(x))$ is the weighted degree of $h(x)$ and $D_{e}(h(x)) \leq m$.

Since the total number of multiplicative characters $\chi^{(d)}$ is $\varphi(d)$, we get

$$
N \geq \delta\left\{\left(q^{n}-1\right)-2^{\omega\left(q^{n}-1\right)}(W-1) m q^{\frac{n}{2}}\right\}
$$

where $\omega\left(q^{n}-1\right)$ is the number of the distinct prime factors of $q^{n}-1$.

Lemma 17. Let $m=p^{e-1} m_{1},\left(m_{1}, p\right)=1$. Let

$$
\theta_{m_{1}, e}=\sum_{l=0}^{e-1}(e-l) \# S_{l}
$$

where $S_{l}$ are defined by (7) and (8)). Then

$$
\theta_{m_{1}, e}=m-\left[\frac{m_{1}}{p}\right],
$$

so $\theta_{m_{1}, e}=m$ if and only if $m_{1}<p$.

Proof. By definition of $S_{l}$, we have

$$
\begin{aligned}
\# S_{0} & =m_{1}-\left[\frac{m_{1}}{p}\right] \\
\# S_{1} & =m_{1} p-m_{1}-\# S_{0} \\
& =m_{1} p-2 m_{1}+\left[\frac{m_{1}}{p}\right]
\end{aligned}
$$

if $1<l<e$, then

$$
\begin{aligned}
\# S_{l} & =m_{1} p^{l}-m_{1} p^{l-1}-\left(m_{1} p^{l-1}-m_{1} p^{l-2}\right) \\
& =m_{1} p^{l}-2 m_{1} p^{l-1}+m_{1} p^{l-2}
\end{aligned}
$$


It is easily check that Lemma 17 holds for $e \leq 2$. Let $e>2$; we use induction on e. Therefore

$$
\begin{aligned}
\theta_{m_{1}, e+1}= & \sum_{l=0}^{e}(e+1-l) \# S_{l} \\
= & \sum_{l=0}^{e-1}(e-l) \# S_{l}+\sum_{l=0}^{e} \# S_{l} \\
= & \theta_{m_{1}, e}+m_{1}-\left[\frac{m_{1}}{p}\right]+m_{1} p-2 m_{1}+\left[\frac{m_{1}}{p}\right] \\
& +\sum_{l=2}^{e}\left(m_{1} p^{l}-2 m_{1} p^{l-1}+m_{1} p^{l-2}\right) \\
= & \theta_{m_{1}, e}-m_{1} p^{e-1}+m_{1} p^{e}
\end{aligned}
$$

and $m_{1} p^{e}-\theta_{m_{1}, e+1}=m_{1} p^{e-1}-\theta_{m_{1}, e}$. By induction hypothesis on $e$, that is, $m_{1} p^{e-1}-\theta_{m_{1}, e}=\left[\frac{m_{1}}{p}\right]$, we have $\theta_{m_{1}, e+1}=m_{1} p^{e}-\left[\frac{m_{1}}{p}\right]$. The second conclusion is obvious. So we finish the proof of Lemma 17.

Theorem 18. Let $N$ be the number of primitive element solutions of (3) in $\Gamma_{n k}^{*}$. For $0<m<n$, let $m=p^{e-1} m_{1},\left(m_{1}, p\right)=1$. If

$$
q^{\frac{n}{2}-\left(m-\left[\frac{m_{1}}{p}\right]\right)}>m 2^{\omega\left(q^{n}-1\right)}
$$

where $\omega\left(q^{n}-1\right)$ is the number of the distinct prime factors of $q^{n}-1$, then $N>0$.

Proof. By (91) and Lemma [17 it is easily seen that $N>0$ if

$$
\begin{aligned}
q^{\frac{n}{2}} & >m 2^{\omega\left(q^{n}-1\right)} q^{\sum_{l=0}^{e}(e-l) \# S_{l}} \\
& =m 2^{\omega\left(q^{n}-1\right)} q^{m-\left[\frac{m_{1}}{p}\right]} .
\end{aligned}
$$

Hence our theorem holds.

Following the method introduced by Lenstra and Schoof [14], for given positive integer $n$ and prime number $p$, if $m-\left[\frac{m_{1}}{p}\right]<\frac{n}{2}$, inequality (10) holds for $q$ large enough, where $q$ is the power of $p$. Therefore we prove

Proposition 19. Let $n$ be a positive integer, $p$ a prime number and $q$ a power of p. For $0<m<n$, let $m=p^{e-1} m_{1},\left(m_{1}, p\right)=1$. If $m-\left[\frac{m_{1}}{p}\right]<\frac{n}{2}$, there exists a constant $c(n, m, p)$ depending on $n, m, p$ such that there exists a primitive polynomial over $F_{q}$ of degree $n$ with the $m$-th coefficient fixed in advance if $q>c(n, m, p)$.

Similary we have

Proposition 20. Let $n$ be a positive integer. If $m<\frac{n}{2}$, there exists a constant $c(n, m)$ depending on $n, m$ such that there exists a primitive polynomial over $F_{q}$ of degree $n$ with the $m$-th coefficient fixed in advance if $q>c(n, m)$.

From Proposition 20, for large enough $q$, the Hansen-Mullen conjecture is true for those terms $x^{n-m}$ such that $m \leq \frac{n}{2}$ except when $m=\frac{n}{2}$ if $n$ is even. But for the terms $x^{n-m}$ such that $m>\frac{n}{2}$, the above estimate (10) gives no information in most cases. However, we can consider the reciprocal polynomial of a primitive polynomial since the reciprocal polynomial of a primitive polynomial is still a primitive polynomial. We hope that we can prove the Hansen-Mullen conjecture of the 
$m$-th coefficient for $m>\frac{n}{2}$ in most cases if $q$ is large enough. In this way we can establish the Hansen-Mullen conjecture of the $m$-th coefficient with only possibly one or two exceptions, that is, those terms $x^{n-m}$ such that $m$ is around $\frac{n}{2}$. In fact, the $(n-m)$-th coefficient can be fixed if the $m$-th coefficient and the constant term can be fixed at the same time.

For this reason, we estimate the number of primitive element solution of (4), which we denote by $N^{\prime}$.

Let $\psi, \psi_{e, n}, \chi^{(d)}$ be defined as before, and let $\chi_{e, n}$ be the multiplicative character over $\Gamma_{n k}$ defined by $\chi_{e, n}=\chi \cdot$ Norm. We have

$$
\begin{aligned}
N^{\prime}= & \delta \sum_{\xi \in \Gamma_{n k}^{*}}\left(\prod_{l=0}^{e-1} \prod_{\substack{t \in S_{l} \\
t \neq m_{1}}} \sum_{c_{t} \in R_{e-l, k}} \psi\left(p^{l} c_{t} \operatorname{Tr}\left(\xi^{t}\right)\right)\right. \\
& \times\left(\sum_{c_{m_{1}} \in R_{e, k}} \psi\left(c_{m_{1}}\left(\operatorname{Tr}\left(\xi^{m_{1}}\right)-p^{e-1} a\right)\right)\right) \\
& \times \sum_{\chi} \chi\left(\frac{N o r m(\xi)}{b}\right) \sum_{d \mid q^{n}-1} \frac{\mu(d)}{\varphi(d)} \sum_{\chi^{(d)}} \chi^{(d)}(\xi) \\
= & \delta \sum_{\chi} \chi\left(\frac{1}{b}\right) \sum_{d \mid q^{n}-1} \frac{\mu(d)}{\varphi(d)} \sum_{\chi^{(d)}} \sum_{\left(c_{t}\right)_{(t, p)=1} \in \mathbb{S}} \psi\left(-p^{e-1} c_{m_{1}} a\right) \\
& \times \Lambda\left(\left(c_{t}\right)_{(t, p)=1}, \chi^{(d)}, \chi_{e, n}\right) .
\end{aligned}
$$

In the above, $\chi$ run across all the multiplicative characters over $T_{k}^{*}$,

$$
\delta=\frac{1}{(q-1)} \frac{\varphi\left(q^{n}-1\right)}{\left(q^{n}-1\right)} q^{-\sum_{l=0}^{e-1}(e-l) \# S_{l}}
$$

and

$$
\Lambda\left(\left(c_{t}\right)_{(t, p)=1}, \chi^{(d)}, \chi_{e, n}\right)=\sum_{\xi \in \Gamma_{n k}^{*}}\left(\chi^{(d)} \cdot \chi_{e, n}\right)(\xi) \psi_{e, n}(h(\xi))
$$

where $\left(c_{t}\right)_{(t, p)=1} \in \mathbb{S}$ and $h(x)$ is defined as before.

Now we estimate $N^{\prime}$.

(1) Suppose $h(x) \neq 0$. Then $c_{t} \neq 0$ for some $t \in S_{l}$ and some $l$ such that $0 \leq l \leq e-1$. From Theorems 5 and 6 ,

$$
\left|\Lambda\left(\left(c_{t}\right)_{(t, p)=1}, \chi^{(d)}, \chi_{e, n}\right)\right| \leq D_{e}(h(x)) \cdot q^{\frac{n}{2}},
$$

and since the number of the multiplicative characters with order $d$ is $\varphi(d)$,

$$
\begin{aligned}
& \left|\sum_{\chi} \chi\left(\frac{1}{b}\right) \sum_{d \mid q^{n}-1} \frac{\mu(d)}{\varphi(d)} \sum_{\chi^{(d)}} \sum_{h(x) \neq 0} \psi\left(-p^{e-1} c_{m_{1}} a\right) \cdot \Lambda\left(\left(c_{t}\right)_{(t, p)=1}, \chi^{(d)}, \chi_{e, n}\right)\right| \\
& \quad \leq(q-1) \cdot 2^{\omega\left(q^{n}-1\right)} \cdot\left(q^{m-\left\lfloor\frac{m_{1}}{p}\right\rfloor}-1\right) \cdot D_{e}(h(x)) \cdot q^{\frac{n}{2}} .
\end{aligned}
$$

(2) Suppose $h(x)=0$. Then $c_{t}=0 \in R_{e-l, k}$ for $t \in S_{l}$, where $l=0,1, \cdots, e-1$.

(a) When $\chi^{(d)} \cdot \chi_{e, n}=\chi_{0}$,

$$
\psi\left(p^{e-1} c_{m_{1}} a\right)=1
$$

and

$$
\Lambda\left(\left(c_{t}\right)_{(t, p)=1}, \chi^{(d)}, \chi_{e, n}\right)=q^{n}-1 .
$$


Let $\chi_{e, n}^{-1}$ be the multiplicative character such that $\chi_{e, n}^{-1} \cdot \chi_{e, n}=\chi_{0}$; we have $\operatorname{order}(\chi)=\operatorname{order}\left(\chi_{e, n}\right)=\operatorname{order}\left(\chi_{e, n}^{-1}\right)$. Let $\chi_{\left(d_{1}\right)}$ be the multiplicative character $\chi$ over $\Gamma_{k}$ with order $d_{1}$; we have

$$
\begin{aligned}
& \sum_{\chi} \chi\left(\frac{1}{b}\right) \sum_{d \mid q^{n}-1} \frac{\mu(d)}{\varphi(d)} \sum_{\chi_{e, n} \cdot \chi^{(d)}=\chi_{0}} \sum_{h(x)=0} \psi\left(-p^{e-1} c_{m_{1}} a\right) \cdot \Lambda\left(\left(c_{t}\right)_{(t, p)=1}, \chi^{(d)}, \chi_{e, n}\right) \\
& =\sum_{d_{1} \mid q-1} \frac{\mu\left(d_{1}\right)}{\varphi\left(d_{1}\right)} \sum_{\chi_{\left(d_{1}\right)}} \chi_{\left(d_{1}\right)}\left(\frac{1}{b}\right)\left(q^{n}-1\right)=\frac{q-1}{\varphi(q-1)}\left(q^{n}-1\right)
\end{aligned}
$$

where the last equation holds since $\frac{1}{b}$ is a primitive element.

(b) When $\chi^{(d)} \cdot \chi_{e, n} \neq \chi_{0}$,

$$
\Lambda\left(\left(c_{t}\right)_{(t, p)=1}, \chi^{(d)}, \chi_{e, n}\right)=0
$$

and

$$
\begin{array}{r}
\sum_{\chi} \chi\left(\frac{1}{b}\right) \sum_{d \mid q^{n}-1} \frac{\mu(d)}{\varphi(d)} \sum_{\chi e, n} \chi_{\chi^{(d)} \neq \chi_{0}} \sum_{h(x)=0} \psi\left(-p^{e-1} c_{m_{1}} a\right) \\
\cdot \Lambda\left(\left(c_{t}\right)_{(t, p)=1}, \chi^{(d)}, \chi_{e, n}\right)=0 .
\end{array}
$$

Therefore

$$
N^{\prime} \geq \delta \cdot\left(\frac{q-1}{\varphi(q-1)}\left(q^{n}-1\right)-(q-1) \cdot 2^{\omega\left(q^{n}-1\right)} \cdot\left(q^{m-\left\lfloor\frac{m_{1}}{p}\right\rfloor}-1\right) \cdot D_{e}(h(x)) \cdot q^{\frac{n}{2}}\right) .
$$

If

$$
q^{\frac{n}{2}-\left(m-\left\lfloor\frac{m_{1}}{p}\right\rfloor\right)}>\varphi(q-1) \cdot m \cdot 2^{\omega\left(q^{n}-1\right)},
$$

even stronger, if

$$
q^{\frac{n}{2}-\left(m-\left\lfloor\frac{m_{1}}{p}\right\rfloor\right)-1}>m \cdot 2^{\omega\left(q^{n}-1\right)},
$$

we have $N^{\prime}>0$. So we have

Theorem 21. Let $N^{\prime}$ be the number of primitive element solutions of (4) in $\Gamma_{n k}^{*}$. For $0<m<n$, let $m=p^{e-1} m_{1},\left(m_{1}, p\right)=1$. If

$$
q^{\frac{n}{2}-\left(m-\left[\frac{m_{1}}{p}\right]\right)-1}>m \cdot 2^{\omega\left(q^{n}-1\right)}
$$

where $\omega\left(q^{n}-1\right)$ is the number of the distinct prime factors of $q^{n}-1$, then $N^{\prime}>0$.

Again by the method of Lenstra and Schoof, for given $n$ and prime number $p$, if $m-\left[\frac{m_{1}}{p}\right]<\frac{n}{2}-1$, there exists a constant $c(n, m, p)$ depending on $n, m, p$ such that there exists a primitive polynomial over $F_{q}$ of degree $n$ with the $m$-th coefficient fixed as any element in $F_{q}$ and the constant term fixed as any primitive element in $F_{q}$ in advance for $q>c(n, m, p)$, where $q$ is the power of $p$. Considering the reciprocal polynomial of primitive polynomial, we can give the following

Proposition 22. Let $n$ be a positive integer, $p$ a prime number and $q$ power of $p$. For $0<m<n$, if $\left(m-\left\lfloor\frac{m_{1}}{p}\right\rfloor\right)<\frac{n}{2}-1$, there is a constant $c(n, m, p)$ depending on $n, m, p$ such that there exists a primitive polynomial over $F_{q}$ of degree $n$ with the $(n-m)$-th coefficient fixed in advance if $q>c(n, m, p)$. 
We can also get

Proposition 23. Let $n$ be a positive integer and $\frac{n}{2}<m<n$. If $\frac{n}{2}+1<m$, there exists a constant $c(n, m)$ depending on $n, m$ such that there exists a primitive polynomial over $F_{q}$ of degree $n$ with the $m$-th coefficient fixed in advance if $q>$ $c(n, m)$.

Now we give our two main results of this paper.

Theorem 24. Let $n$ be a positive integer, $p$ a prime number and $q$ a power of $p$. Let $n_{1}$ be the "non- $p$ part" of $\frac{n}{2}$ if $n$ is even and the "non- $p$ part" of $\frac{n-1}{2}$ if $n$ is odd. There exists a constant $c(n, p)$ depending on $n, p$ such that the Hansen-Mullen conjecture over $F_{q}$ of the $m$-th $(0<m<n)$ coefficient of primitive polynomial with degree $n$ is true for $q>c(n, p)$ except when:

(1) $m=\frac{n+1}{2}$ if $n$ is odd, $n_{1}<p$;

(2) $m=\frac{n}{2}, \frac{n}{2}+1$ if $n$ is even, $n_{1}<p$.

Proof. We first prove the case of odd $n$. From Proposition 19 there exists a constant $c(n, m, p)$ such that for every $1 \leq m<\frac{n}{2}$ there exists a primitive polynomial of degree $n$ with the $m$-th coefficient over $F_{q}$ if $q>c(n, m, p)$. By Proposition [22, for every pair $(n, m, p)$ such that $\frac{n}{2}<m<n$ when $n_{1} \geq p$ and $\frac{n}{2}+1<m \leq n-1$ when $n_{1}<p$, there exists a constant $c(n, m, p)$ such that there exists a primitive polynomial over $F_{q}$ of degree $n$ with the $m$-th coefficient fixed as any element in $F_{q}$ if $q>c(n, m, p)$. If $n_{1}<p$, let

$$
c(n, p)=\max _{\substack{0<m<n \\ m \neq \frac{n+1}{2}}} c(n, m, p)
$$

and if $n_{1}>p$, let

$$
c(n, p)=\max _{0<m<n} c(n, m, p) .
$$

Then the Hansen-Mullen conjecture over $F_{q}$ of the $m$-th $(0<m<n)$ coefficient of primitive polynomial with degree $n$ is true for $q>c(n, p)$ except when $m=\frac{n+1}{2}$ if $n$ is odd and $n_{1}<p$.

The proof of even $n$ is similar. So we have completed the proof.

We give still another theorem:

Theorem 25. Let $n$ be a positive integer. There is a constant $c(n)$ depending on $n$ such that there exists a primitive polynomial of degree $n$ over $F_{q}$ with the $m$-th $(0<m<n)$ coefficient fixed in advance for $q>c(n)$ except when $m=\frac{n+1}{2}$ if $n$ is odd and when $m=\frac{n}{2}, \frac{n}{2}+1$ if $n$ is even.

Proof. From Propositions 20] and 23] we can easily obtain the proof.

\section{ACKNOWLedgement}

The authors are indebted to the referee for her or his valuable comments which corrected several errors in the original paper and made the paper simpler and more succinct. 


\section{REFERENCES}

1. S.D.Cohen, Primitive elements and polynomials with arbitrary traces, Discrete Math, vol. 83, no. 1, pp. 1-7, 1990. MR 91h:11143

2. S.D.Cohen, Primitive elements and polynomials: existence results, Lect. Notes in Pure and Applied Math, vol. 141, edited by G.L.Mullen and P.J.Shiue, Dekker, New York, pp. 43-55, 1993. MR 93k:11113

3. H.Davenport, Bases for finite fields, J.London Math. Soc, vol. 43, pp. 21-39, 1968. MR 37:2729

4. B. Dwork, G.Gerotto and F.J.Sullivan, An Introduction to G-Functions, Annals of Mathematics Studies, Number 133, Princeton University Press, 1994. MR 96c:12009

5. W-B.Han, The coefficients of primitive polynomials over finite fields, Math. of Comp, vol. 65, no. 213, pp. 331-340, Jan. 1996. MR 96d:11128

6. W-B.Han, On two exponential sums and their applications, Finite Fields and Their Applications, 3, pp. 115-130, 1997.

7. W-B.Han, On Cohen's Problem, Chinacrypt'96, Academic Press(China), pp. 231-235, 1996 (in Chinese).

8. W-B.Han, The distribution of the coefficients of primitive polynomials over finite fields, Proceeding of CCNT'99, Prog.in Comp. Sci. and Applied. Logic, vol. 20, Birkhäuser Verlag, Basel/Switzerland, pp. 43-57, 2001.

9. T.Hansen and G.L.Mullen, Primitive polynomials over finite fields, Math. of Comp, vol. 59, no. 200, pp. 639-643, Supplement: S47-S50, Oct. 1992. MR 93a:11101

10. T.Helleseth, P.V.Kumar, O.Moreno and A.G.Shanbhag, Improved estimates via exponential sums for the minimum distance of $\mathbb{Z}_{4}$-linear trace codes, IEEE. Trans. Inform. Theory, vol. 42, no.4, pp. 1212-1216, July 1996. MR 97m:94028

11. D.Jungnickel and S.A.Vanstone, On primitive polynomials over finite fields, J. of Algebra, vol. 124, pp. 337-353, 1989. MR 90k:11164

12. N. Koblitz, p-adic number, $p$-adic analysis and zeta functions, GTM58, Springer-Verlag, 1984. MR 86c:11086

13. P.V.Kumar, T.Helleseth and A.R.Calderbank, An upper bound for Weil exponential sums over Galois rings and applications, IEEE. Trans. Inform. Theory, vol. 41, no.2, pp. 456-468, Mar. 1995. MR 96c:11140

14. H.W.Lenstra and R.J.Schoof, Primitive normal bases for finite fields, Math. of Comp, vol. 48, no. 177 , pp. 217-232, 1987. MR 88c:11076

15. W.-C.W.Li, Character sums over $p$-adic fields, J. Number Theory, 74, pp. 181-229, 1999. MR 2000b: 11100

16. R.Lidl, H.Niedereiter, Finite Fields, Addison-Wesley, London, 1983. MR 86c:11106

17. O.Moreno, On the existence of a primitive quadratic trace over $G F\left(p^{m}\right)$, J. of Combin. Theory Ser. A, vol. 51, pp. 104-110, 1989. MR 90b:11133

Department of Applied Mathematics, Information Engineering University, Zhengzhou, 450002, People's Republic of China

E-mail address: sq.fan@263.net

Department of Applied Mathematics, Information Engineering University, ZhengZHOU, 450002, People's Republic of China

E-mail address: wb.han@netease.com 